\title{
The enclosed ward management strategies in psychiatric hospitals during COVID-19 outbreak
}

\author{
Jiajia Chen ${ }^{1,2^{*}} \mathbb{D}$, Maoxiang Xiong ${ }^{1,2+}$, Zongling He $e^{1,2}$, Wen Shi ${ }^{1,2}$, Yuchuan Yue ${ }^{1,2}$ and Manxi He $e^{1,2^{*}}$
}

\begin{abstract}
During the COVID-19 pandemic, as a large city located in Southwest China, Chengdu was mainly affected by imported cases. For a psychiatric hospital, the enclosed management model, the crowded wards and the uncooperative patients are the risk factors of nosocomial infection. Admitting new patients while preventing the COVID-19 outbreak within the institutions was a crucial challenge. The Mental Health Centre of Chengdu proposed a series of effective management strategies to deal with the rapidly evolving situation during the COVID-19 pandemic which included regulation for the inpatients, their families and staff, and achieved Zero infection in our hospital.
\end{abstract}

Keywords: Psychiatric hospital, COVID-19 pandemic, Enclosed wards, Management strategies

In December 2019, novel coronavirus pneumonia (also called COVID-19) emerged in Wuhan and soon spread to other large cities in China. In early February 2020, due to nosocomial infection, 80 patients and staff from the Wuhan Mental Health Centre were diagnosed with COVID-19 [1], whereas 119 people were confirmed to be infected in Daenam Hospital, South Korea, in early March [2]. For psychiatric hospitals, the enclosed management model, crowded wards and uncooperative patients are risk factors for hospital-associated infection [3]. Admitting new mental health patients while preventing hospital infection was a crucial challenge. The largest mental health center in southwestern China, the Mental Health Centre of Chengdu, adopted a series of effective management strategies to address the rapidly evolving situation and successfully achieved the goal of zero infection. Our 5 stages of coping strategies followed the timeline of the pandemic, as described below.

\footnotetext{
* Correspondence: 17570003@qq.com; 2357622298@qq.com

†iajia Chen and Maoxiang Xiong contributed equally to this work.

'The Clinical Hospital of Chengdu Brain Science Institute, School of Life

Science and Technology, University of Electronic Science and Technology of China, Chengdu, Sichuan, China

Full list of author information is available at the end of the article
}

\section{Stage one}

At the beginning of the outbreak (mid-January 2020), we immediately formed a COVID-19 prevention and control leadership group and established enclosed ward management regulations, such as temporarily stopping inpatient admission and prohibiting visits (families contacted patients via video call instead) [4]. In each ward, we set up two observation rooms for inpatients, in case they had fever or other respiratory symptoms. Specific regulations were formulated for patients' relatives, caregivers and janitors, requiring them to stay in the wards $24 \mathrm{~h} /$ day and 7 days/week. All the food and drinks were served by the canteen, where the staff's health condition was carefully monitored. No take-away food was allowed.

\section{Stage two}

At the end of January 2020, the COVID-19 epidemic was expanding all over China. The Chengdu local government required all the public hospitals to help treat diagnosed cases and suspected cases. Our hospital was a designated hospital for suspected cases, which increased our risk of COVID-19 exposure. We promptly set up an isolation ward for suspected cases on the top floor of the inpatient building, which 
was a former clinical trial lab with an individual sewer system and air-purifying system, and assembled the top doctors of our hospital to form the medical team. To avoid cross infection, we optimized a special route to the isolation ward and disabled all the air-conditioners. All staff and their families had to follow specific regulations, which required them to report their personal health condition and whereabouts daily. They were also advised to stay home after work, avoid gatherings or leaving Chengdu, and try not to take any public transport -a transport fund was given as a financial support.

\section{Stage three}

In mid-February 2020, the COVID-19 pandemic reached its peak in China. We were at the patient intake peak of the year. To meet the substantial demand for hospitalization, we set up a transitional observation ward with 60 beds. In the observation ward, new patients were observed in separated rooms for 14 days [3] until the relevant standards were met, at which point they were transferred to a regular ward. Staff who worked in this ward could not return home until the patients were confirmed to be noninfectious. If a staff member had close contact with a confirmed case, then they would have to go through a 14-day observation and COVID-19 nucleic acid test.

\section{Stage four}

At the end of February, the pandemic remained at the peak. To avoid cross infection, we placed new patients with and without fever into two separate areas. We mobilized a ward with 40 beds for the suspected patients with fever called the "fever ward". As a supplement for the observation ward, we enacted total physical isolation for different patients. At that point, our hospital formulated a mature procedure for patient transfer from the fever ward/observation ward to the regular ward.

\section{Stage five}

At the end of March, as the pandemic eased in China, there were no new cases reported in Chengdu for several consecutive days. We disassembled the observation wards while maintaining the whole manpower structure. Instead of observation wards, in each regular ward, we installed two areas: one was a "fever area", and the other was an observation area. New patients were assigned to these two areas according to their physical condition. COVID-19 nucleic acid examination and chest $\mathrm{CT}$ scan were performed among all new patients to eliminate the risk of carrying the novel coronavirus. After 14 days of observation, if certain criteria (negative nucleic test result, negative chest $\mathrm{CT}$ result and normal body temperature for 3 days) [5] were met, these new patients were transferred to general rooms.

As the understanding of COVID-19 is constantly updated, we have adjusted our prevention strategies in a timely manner. When the pandemic situation improved, we suggested that staff could leave Chengdu but not $\mathrm{Si}$ chuan Province; otherwise, they will have to go through 14-day self-quarantine and COVID-19 nucleic acid tests. In the summer, we will fix separate air-conditioners with air-purifying functions in each ward.

As stated above, psychiatric hospitals are facing critical administrative challenges in this pandemic. We hope that our experiences can serve as a reference for other mental health hospitals in terms of minimizing the adverse outcomes of this pandemic for the mentally ill population.

\section{Acknowledgements \\ The Authors wish to thank all the clinical staff of the Mental Health Centre of Chengdu.}

\section{Authors' contributions \\ Jiajia Chen and Maoxiang Xiong read and screened articles for inclusion, sorted out measures and standards, summed up practical experiences and drafted the manuscript. Zongling He, Wen Shi and Yuchuan Yue reviewed the work measures, standards and practical experience, and offered a proposal. Manxi He reviewed the whole article and revised it according to the standards. The authors read and approved the final manuscript.}

Funding

None.

Availability of data and materials

Not applicable.

Ethics approval and consent to participate

Not applicable.

Consent for publication

Not applicable.

\section{Competing interests}

The authors declare that they have no competing interests.

\section{Author details}

${ }^{1}$ The Clinical Hospital of Chengdu Brain Science Institute, School of Life Science and Technology, University of Electronic Science and Technology of China, Chengdu, Sichuan, China. ${ }^{2}$ The Mental Health Centre of Chengdu, Sichuan 610036, China.

Received: 28 April 2020 Accepted: 17 June 2020

Published online: 24 June 2020

References

1. Yutao X, Yanjie Z, Zihan L. The COVID-19 outbreak and psychiatric hospital in China: managing challenges through mental health service reform. Int J Biol Sci. 2020;16(10):1741-4. https://doi.org/10.7150/ijbs.45072.

2. Ji H, Li L, Huang T, Zhu Y. Nosocomial infections in psychiatric hospitals during the new coronavirus pneumonia outbreak. Eur J Psychiatry. 2020. https://doi.org/10.1016/j.ejpsy.2020.04.001.

3. Yucheng $Z$, Liangliang C, Haifeng J. The risk and prevention of Novel Coronavirus Pneumonia infections among inpatients in psychiatric hospitals. Neurosci Bull. 2020;36(3):299-302. https://doi.org/10.1007/s12264-020-00476-9.

4. Ying S, Yang S, Jianming F. Psychiatry hospital management facing COVID19: from medical staff to patients. Brain Behav Immun. 2020. https://doi.org/ 10.1016/j.bbi.2020.04.018

5. National Health Commission of China, Guidelines for the diagnosis and treatment of novel coronavirus pneumonia (in Chinese). http//mww.nhc.gov.cn/yzygj/s7653p/202 003/46c9294a7dfe4cef80dc7f5912eb1989.shtml. Accessed 4th March 2020.

\section{Publisher's Note}

Springer Nature remains neutral with regard to jurisdictional claims in published maps and institutional affiliations. 